02

\title{
Особенности частотной дисперсии естественной оптической активности неидеального молекулярного кристалла в экситонной области энергетического спектра
}

\author{
(C) А.Е. Рыбалка, В.В. Румянцев, С.А. Федоров, К.В. Гуменник
}

Донецкий фризико-технический институт им. А.А. Галкина, 83114 Донецк, Украина

e-mail: rybalka96@inbox.ru

Поступила в редакцию 11.03.2021 г.

В окончательной редакции 22.03.2021 г.

Принята к публикации 24.03.2021 г.

Рассмотрены особенности естественной оптической активности неидеального молекулярного кристалла и выполнено численное моделирование ее частотной дисперсии в экситонной области энергетического спектра. Исследована зависимость вращательной способности от концентрации структурных дефектов в данной кристаллической решетке для различных частотных интервалов.

Ключевые слова: неидеальная кристаллическая структура, естественная оптическая активность, экситонный резонанс.

DOI: $10.21883 / O S .2021 .07 .51078 .1994-21$

\section{Введение}

Исследованию оптических свойств кристаллических систем в экситонной области спектра посвящено достаточно много работ [1-5]. Они позволяют глубже понять электронную структуру кристаллов, свойства квазичастиц и взаимодействие между ними. В результате экспериментального изучения экситонов в совершенных структурах при низких температурах выявлены существенные особенности спектров экситонов. При этом, как справедливо отмечает С.И. Пекар [2], при изучении оптических явлений в кристаллах особенности, связанные с сильным взаимодействием структурных единиц кристалла с электромагнитным полем („поляритонный эффект") следует отличать от явления пространственной дисперсии (ПД), т. е. зависимости диэлектрической проницаемости $\widehat{\varepsilon}$ как от частоты $\omega$, так и от волнового вектора $\mathbf{k}$ электромагнитной волны. Поляритонному эффекту уделяется значительное внимание [5], начиная с классических моделей М. Борна (1915 г.) в динамической теории кристаллических решеток, в то время как существенные проявления ПД света в кристаллах были обнаружены только в 1957 г. [2,6]. Термин „пространственная дисперсия“, констатирующий зависимость $\hat{\varepsilon}(\mathbf{k})$, был введен М.Е. Герценштейном в 1952 г. [7].

Учет ПД дает возможность расширить спектр изучаемых явлений и рассматривать такие эффекты, как естественная оптическая активность (ЕОА), электрогирация, круговой дихроизм и т. . Изучение дисперсии гиротропных характеристик позволяет выявлять структурные особенности соответствующих сред, определять важные динамические параметры образующих их молекул $[8,9]$, а также помогает учитывать эффекты ПД в поляризационных измерениях спектральных харак- теристик нелинейных оптических процессов. Гиротропия часто является единственно возможным способом определения некоторых стерео- и кристаллохимических параметров хиральных систем, а также тонких деталей строения соответствующих пространственно диспергирующих структур. Такая характеристика, как молекулярная хиральность, представляет особый интерес для биохимических и фармакологических исследований многих биологических систем. Примерами хиральных систем могут служить аминокислоты, сахара, белки и ферменты. В то время как биомолекулы в основном были изучены в растворах, большое значение в фармацевтике имеет изучение свойств их твердотельной фазы.

Реальные кристаллические системы всегда содержат структурные дефекты, поэтому интерпретация экспериментальных данных и выявление соответствующих механизмов гиротропии, а также понимание их особенностей требуют развития микротеории, учитывающей указанные несовершенства. В настоящей работе на основе экситонной теории ЕОА молекулярных кристаллов [10] выполнено численное моделирование зависимости важной количественной характеристики гиротропии - вращательной способности - неидеального тернарного молекулярного кристалла от концентрации дефектов структуры и ее частотной дисперсии в экситонной области спектра

\section{Теоретическая модель}

Известно, что ответы на ряд вопросов таких, как определение частотной дисперсии угла вращения плоскости поляризации линейно поляризованной монохроматической электромагнитной волны, нахождение связи 
угла вращения со структурой экситонных состояний, определение численных значений исследуемых величин и т.п., могут быть получены лишь в рамках микроскопической теории ЕОА. Применительно к молекулярным кристаллам построение микротеории ЕОА оказалось возможным после создания Аграновичем [10] и Давыдовым [11] теории экситонов Френкеля для молекулярных кристаллов. В работе [10] при исследовании EOA экситонные состояния рассматривались в рамках приближения Гайтлера-Лондона (Г-Л) без учета смешивающего межмолекулярного взаимодействия. Последнее было сделано в работе [12] в первом порядке теории возмущений. Теория ЕОА кристаллов, не основывающаяся на приближении Г-Л развивалась в работе [13], но в ней рассматривались лишь одноосные кристаллы, не учитывалось смешивание молекулярных состояний и игнорировалась зависимость волновых функций экситонов от волнового вектора k. И, наконец, в выше перечисленных работах $[10,12,14]$ производные по волновому вектору от экситонных характеристик (энергий и волновых функций) не связывались с конкретной структурой кристалла (микрохарактеристики среды), а фигурировали в качестве независимых параметров теории. В результате выражение для тензора гиротропии и вращательной способности были громоздкими и мало удобными для анализа различных экспериментальных ситуаций. Микроскопическое рассмотрение ЕОА, свободное от указанных недостатков, проведено авторами $[15,16]$. В настоящей работе используется именно эта микротеория.

Экситонная теория оптической активности молекулярных кристаллов $[15,16]$ дает возможность определить важную количественную характеристику гиротропии - вращательную способность идеального кристалла $\rho(\mathbf{s}, \omega)$. Используя формулу (6) из [16] и выражение (6.13) из [10], получим следующее выражение для $\rho(\mathbf{s}, \omega)$ :

$$
\begin{aligned}
\rho(\mathbf{s}, \omega)= & \frac{2 \pi \omega^{2}}{v_{0} c^{2}} s^{t}\left\{\frac{2 h}{i} \sum_{\mu} \frac{Q_{s \mu: 0}^{t l}\left[\mathbf{s} \times \mathbf{P}_{0: s \mu}\right]^{l}}{E_{\mu}^{2}(\mathbf{s})-\hbar^{2} \omega^{2}}\right. \\
& \left.+\sum_{\mu \nu} \frac{E_{\mu}(\mathbf{s}) E_{v}(\mathbf{s})\left[\mathbf{P}_{0: s \mu} \times \mathbf{P}_{s v: 0}\right] \mathbf{s} W_{\mu \nu}^{t}(\mathbf{s})}{\left[E_{\mu}^{2}(\mathbf{s})-\hbar^{2} \omega^{2}\right]\left[E_{v}^{2}(\mathbf{s})-\hbar^{2} \omega^{2}\right.}\right\} .
\end{aligned}
$$

Здесь $v_{0}$ - объем элементарной ячейки, $\mathbf{s}=\mathbf{k} / k, P_{s \nu 0}^{l}-$ матричный элемент оператора дипольного момента кристалла, вычисленный на волновых функциях экситонов, $E_{\mu}(\mathbf{s}), E_{v}(\mathbf{s})$ - энергии экситонов при $k=0$, соответствующих $\mu$-й и $v$-й экситонным зонам. Величины $Q_{s \mu: 0}^{t l}$ и $W_{\mu \nu}^{t}$ определяются [16] равенствами:

$$
\begin{aligned}
Q_{0 ; s \mu}^{t l}= & \sum_{f \alpha}\left\langle\phi_{\mathbf{n} \alpha}^{(0)}\left|i \partial J_{\mathbf{n} \alpha}^{i}(\mathbf{k}) / \partial k^{t}\right|_{k=0} \mid \phi_{\mathbf{n} \alpha}^{(f)}\right\rangle\left[u_{f \alpha \mu}(\mathbf{s})-v_{f \alpha \mu}(\mathbf{s})\right] \\
& W_{\mu \nu}^{t}(\mathbf{s})=\left\langle\Psi_{s \mu}\left|i \partial H^{(e x)^{2}}(\mathbf{k}) / \partial k^{t}\right|_{k=0} \mid \Psi_{s \nu}\right\rangle
\end{aligned}
$$

где $H^{(e x)}$ - экситонная часть гамильтониана исследуемого кристалла, выделение которой осуществляется путем последовательного применения метода приближенного вторичного квантования к случаю молекулярных экситонов [11]. Согласно этому методу, переход к представлению вторичного квантования производится с помощью определенной системы волновых функций $\phi_{\mathbf{n} \alpha}^{(f)}$ молекул $(f-$ набор соответствующих квантовых чисел), $\Psi_{\mu}(\mathbf{k})$ - волновая функция экситонного состояния $\mu$-й экситонной зоны $(\mathbf{k}-$ волновой вектор экситона в первой зоне Бриллюэна), $\mathbf{J}_{\mathbf{n} \alpha}(\mathbf{k})-$ фурьеобраз оператора плотности тока молекулы $\mathbf{n} \alpha, u_{f \alpha \mu}(\mathbf{s})$, $v_{a \alpha \mu}(\mathbf{s})$ - коэффициенты преобразования БоголюбоваТябликова.

Первое слагаемое в (1) соответствует молекулярной составляющей, а второе - кристаллической составляющей вращательной способности. Для кристаллов с примитивной решеткой второе слагаемое в (1) равно нулю и частотная дисперсия описывается лишь первым слагаемым.

Исследование $\rho(\mathbf{s}, \omega)$ вблизи экситонных резонансов представляет особый интерес, поскольку эта измеряемая характеристика позволяет объяснять частотную дисперсию оптической активности и устанавливать связь экспериментально измеряемого удельного угла вращения с микрохарактеристиками среды.

\section{Вращательная способность неидеальной кристаллической решетки}

Изучим подробнее особенности вращательной способности неидеального молекулярного кристалла и ее частотной дисперсии в экситонной области энергетического спектра в рамках одноуровневой модели, обусловленные наличием дефектов в структуре. Для конкретизации проблемы рассмотрим топологически упорядоченную кристаллическую систему с примитивной кубической решеткой, в узлах которой случайным образом расположены два сорта изотопических молекул и вакансии. В этом случае, как следует из формулы (12) в [16], справедливо равенство

$$
C_{1}+C_{2}+C_{v}=1
$$

где $C_{1}$ и $C_{2}$ - концентрации изотопических молекул соответственно первого (основного) и второго (примесного) сортов, $C_{v}$ - концентрация вакансий.

Для рассматриваемой неидеальной структуры выражение $(1)$ для $\rho(\mathbf{s}, \omega)$ переходит в рассматриваемом случае в соотношение (17) из [16] для $\rho\left(\mathbf{s}, \omega, C_{1}, C_{2}, C_{v}\right)$, которое учитывает зависимость этой величины от концентрации дефектов. Для системы с примитивной решеткой кристаллическая составляющая вращательной способности равна нулю и данное выражение для $\rho\left(\mathbf{s}, \omega, C_{1}, C_{2}, C_{v}\right)$ после несложных преобразований с 

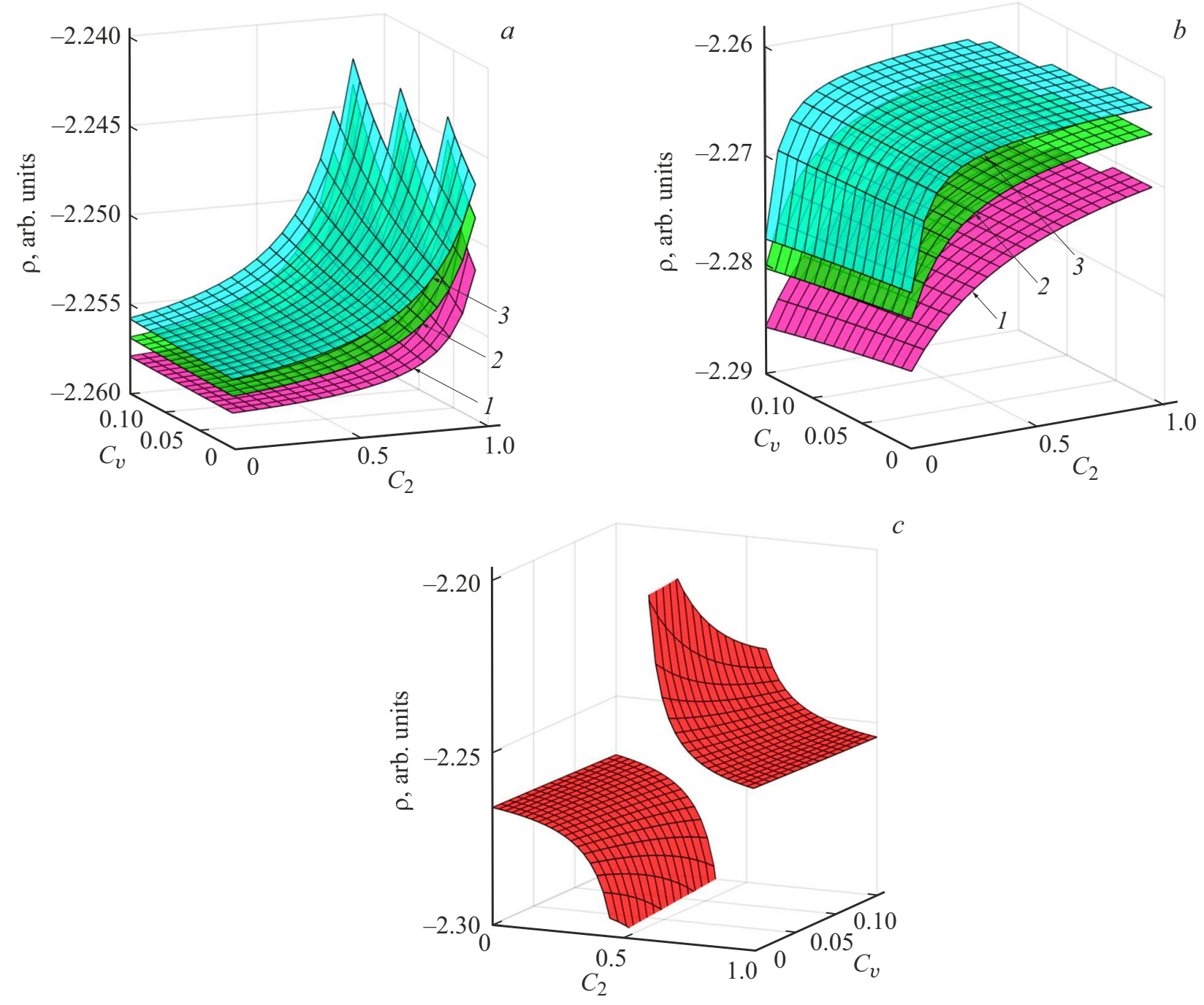

Зависимость вращательной способности от концентрации дефектов структуры в исследуемом неидеальном молекулярном кристалле в экситонной области спектра при $h \omega$, равных: $a-2.9994 \cdot 10^{4}(1), 2.9996 \cdot 10^{4}(2), 2.9998 \cdot 10^{4} \mathrm{~cm}^{-1}(3) ; b-$ $3.0035 \cdot 10^{4}(1), 3.0040 \cdot 10^{4}(2), 3.0050 \cdot 10^{4} \mathrm{~cm}^{-1}(3) ; c-h \omega=3.0015 \cdot 10^{4} \mathrm{~cm}^{-1}$.

учетом (3) приобретает вид

$$
\begin{aligned}
& \rho\left(\mathbf{s}, \omega, C_{1}, C_{2}, C_{v}\right)=\frac{2 A}{\hbar c^{2}}(\hbar \omega)^{2}\left[C_{2}\left(\hbar^{2} \omega^{2}-E_{1}^{2}\right)\right. \\
& +\left(1-C_{2}-C_{v}\right)\left(\hbar^{2} \omega^{2}-E_{2}^{2}\right)-2\left(1-C_{2}-C_{v}\right) \\
& \times C_{2}\left(E_{1} W^{11}+E_{2} W^{22}\right)+\left(1-C_{2}-C_{v}\right) C_{2}\left(W^{12}\right. \\
& \left.\left.+W^{21}\right)\left(E_{1}+e_{2}\right)\right]\left[\left(\hbar^{2} \omega^{2}-E_{1}^{2}\right)\left(\hbar^{2} \omega^{2}-E_{2}^{2}\right)\right. \\
& -2\left(\hbar^{2} \omega^{2}-E_{1}^{2}\right) E_{2} W^{22} C_{2}-2\left(\hbar^{2} \omega^{2}-E_{2}^{2}\right) \\
& \times E_{1} W^{11}\left(1-C_{2}-C_{v}\right)+4 E_{1} E_{2}\left(1-C_{2}-C_{v}\right) \\
& \left.\times C_{2}\left(W^{11} W^{22}-W^{12} W^{21}\right)\right]^{-1} .
\end{aligned}
$$

Здесь $c$ - скорость света, $A=i \frac{2 \pi}{v_{0}}\left[\mathbf{s} \times \mathbf{P}_{0 f}\right]^{l} s^{P} Q_{0 f}^{p l}$, величины $\mathbf{P}_{0 f}$ и $Q_{0 f}^{t l}-$ матрнчные элементы соот- ветственно операторов дипольного момента молекулы и производной по $\mathbf{k}$ оператора фурье-образа плотности тока этой молекулы (для всех изотопических молекул эти величины практически одинаковы), $E_{1}$ и $E_{2}$ - энергии возбуждения молекул соответственно первого и второго сортов, $W^{v \mu}-$ фурье-образ (при $\mathbf{k}=0)$ матрицы резонансного межмолекулярного взаимодействия, соответствующей кулоновскому взаимодействию молекул $v$-го и $\mu$-го сортов. Для изотопических молекул с хорошей точностью можно полагать $W_{11} \approx W_{22} \approx W_{12} \approx W_{21} \approx 0.7 \cdot 10^{4} \mathrm{~cm}^{-1}[17]$.

\section{Результаты и обсуждение}

Значительный интерес при исследовании ЕОА неидеальных систем представляет изучение зависимости вращательной способности в различных частотных областях от концентрации структурных дефектов. В рам- 
ках одноуровневой модели проведем рассмотрение особенностей $\rho\left(\mathbf{s}, \omega, C_{1}, C_{2}, C_{v}\right)$, обусловленных наличием дефектов в структуре топологически упорядоченной кристаллической системы с примитивной кубической решеткой, в узлах которой случайным образом расположены два сорта изотопических молекул и вакансии с концентрациями соответственно $C_{1}, C_{2}$ и $C_{\nu}$. Диапазон изменения значений концентраций $C_{v}$ ограничен условием устойчивости соответствующей кристаллической структуры (конкретный расчет предельной величины $C_{v}$ для каждого отдельного случая определяется типом химической связи, меж- и внутримолекулярным взаимодействием молекул [18]). Полагаем, что для рассматриваемой модельной структуры $C_{v} \in(0,0.15)$.

Для конкретизации проблемы рассмотрим (с учетом $\left.C_{1}=1-C_{2}-C_{\nu}\right)$ вращательную способность $\rho\left(\mathbf{s}, \omega, C_{2}, C_{v}\right)$ модельного молекулярного кристалла со случайной изотопической заменой молекул, например, атомов водорода на атомы дейтерия в структуре бензола в интервале $h \omega$ вблизи типичных [17] для данного соединения молекулярных энергий возбуждения: $E_{1}=3 \cdot 10^{4} \mathrm{~cm}^{-1}, E_{2}=3.0033 \cdot 10^{4} \mathrm{~cm}^{-1}$. Численный расчет зависимости вращательной способности от концентрации дефектов структуры в исследуемом неидеальном молекулярном кристалле в экситонной области энергетического спектра вблизи энергии $E_{1}$ возбуждения молекул при $h \omega$, равных $2.9994 \cdot 10^{4} \mathrm{~cm}^{-1}$, $2.9996 \cdot 10^{4} \mathrm{~cm}^{-1}, 2.9998 \cdot 10^{4} \mathrm{~cm}^{-1}$, или вблизи энергии $E_{2}$, равных $3.0035 \cdot 10^{4} \mathrm{~cm}^{-1}, 3.0040 \cdot 10^{4} \mathrm{~cm}^{-1}$, $3.0050 \cdot 10^{4} \mathrm{~cm}^{-1}$, показывает, что для рассматриваемой системы имеются частотные области, которым соответствуют существенно различные зависимости вращательной способности от $C_{2}, E_{v}$. В частности, из полученных графиков, которые приведены (в относительных единицах) на рисунках $a, b$, следует, что при удалении $h \omega$ от энергий возбуждения $E_{1}, E_{2}$ поверхности $\rho\left(C_{2} C_{v}\right)$ демонстрируют резкий рост при увеличении величины $C_{2}$ при $h \omega<E_{1}$. В то же время при $h \omega>E_{2}$ поверхности $\rho\left(C_{2}, C_{v}\right)$ становятся более пологими, т.е. концентрационная зависимость вращательной способности от структурных дефектов слабеет. С другой стороны, при малых $C_{2}$ при $h \omega<E_{1}$ оптическая активность менее чувствительна к изменению концентрации $C_{2}$, а при $h \omega>E_{2}$ график указывает на резкое убывание $\rho\left(C_{2}, C_{v}\right)$. Что касается роли вакансий в исследуемой системе, для частотной области $h \omega<E_{1}$ ЕОА оказывается более чувствительной к изменению $C_{v}$, чем для $h \omega>E_{2}$. На этот факт указывает и график на рисунке $c$, который отражает поведение функции $\rho\left(\omega, C_{2}, C_{v}\right)$ при значениях $h \omega$ внутри интервала $\left(E_{1}, E_{2}\right)$. Появление сингулярности на графике $\rho\left(\omega, C_{2}, C_{v}\right)$ для определенных значений концентраций $C_{2}, C_{v}$ структурных дефектов свидетельствует о наличии экситонного резонанса в системе.

\section{Заключение}

Выполнено исследование особенностей вращательной способности неидеального молекулярного кристалла и ее частотной дисперсии в экситонной области спектра, обусловленных наличием дефектов структуры. Полученный результат представляет интерес, поскольку он позволяет объяснять частотную дисперсию оптической активности и устанавливать связь экспериментально измеряемого удельного угла вращения с микрохарактеристиками среды. В настоящее время известно большое количество сложных органических комплексов и полимеров, которые являются оптически активными вследствие особенностей структуры или оптической активности входящих в их состав молекул $[8,19]$. Благодаря значительному прогрессу в теории оптических свойств идеальных и неидеальных структур, разнообразию функциональных свойств и широкой области применения исследование подобных композиционных материалов достаточно актуально [19-21].

\section{Финансирование работы}

Работа выполнена в рамках бюджетного финансирования Донецкого физико-технического института им. А.А. Галкина, проект: „Формирование структуры и свойств перспективных многофункциональных материалов“.

\section{Конфликт интересов}

Авторы заявляют, что у них нет конфликта интересов.

\section{Список литературы}

[1] Агранович В.М., Гинзбург В.Л. Кристаллооптика с учетом пространственной дисперсии и теория экситонов. М.: Наука, 1979. 432 с.

[2] Пекар С.И. Кристаллооптика и добавочные световые волны. Киев: Наук. думка, 1982. 296 с.

[3] Бродин М.С., Мясников Э.Н., Марисова С.В. Поляритоны в кристаллооптике. Киев: Наук. думка, 1984. 200 с.

[4] Румянцев В.В. Взаимодействие электромагнитного излучения и легких частиц с несовершенными кристаллическими средами. Донецк: Норд-Пресс, 2006. 347 с.

[5] Polaritons: Proceedings of the First Tacrmina Conference on Structure of Matter / Ed. by Burstein A., De Martini F. NY etc.: Pergamon press, 1974. $391 \mathrm{c}$.

[6] Пекар С.И. // ЖЭТФ. 1957. Т. 33. В. 4. С. 1022-1036.

[7] Гериенштейн М.Е. // ЖЭТФ. 1952. Т. 22. № 3. С. 303-309.

[8] Кизель В.А., Бурков В.И. Гиротропия кристаллов. М.: Наука, 1980. 304 с.

[9] Федоров Ф.И. Теория гиротропии. Минск: Наука и техника, $1976.156 \mathrm{c}$.

[10] Агранович В.М. Теория экситонов. М.: Наука, 1968. 382 с.

[11] Давыдов А.С. Теория молекулярных экситонов. М.: Наука, 1968. $296 \mathrm{c}$. 
[12] Каменоградский Н.Е. // Труды Института экспериментальной и теоретической метрологии. 1976. Т. 4(61). C. $22-34$.

[13] Овандер Л.Н., Тю Н.С., Федоров С.А. // УФЖ. 1983. Т. 28. № 11. C. 1674-1677.

[14] Kato T. // J. Phys. Soc. Jap. 1973. V. 34. P. 763-768.

[15] Rumyantsev V.V., Fedorov S.A., Gumennyk K.V. Theory of Optically Active Imperfect Composite Materials. Selected Topics. Colne: LAP LAMBERT Academic Publishing, 2012. $52 \mathrm{p}$.

[16] Рыбалка А.Е., Румянцев В.В., Федоров С.А. // Мониторинг. Наука и технологии. 2020. Т. 44. № 2. С 79-86.

[17] Броуде В.Л., Рашба Э.И., Шека Е.Ф. Спектроскопия молекулярных кристаллов. М.: Энергоиздат, 1981. 248 с.

[18] Стоунхэм А.М. Теория дефектов в твердых телах. Т. 2. М.: Наука, 1978. 357 с.

[19] Ryan A.J., Jones R.A.L. // Materials Today. 2008. V. 11. N 7-8. P. 21-23.

[20] Chun Zhang, Hirt D.E. // Polymer. 2007. V. 48. N 23. P. 6748-6754.

[21] Rumyantsev V.V., Fedorov S.A., Proskurenko M.V. // J. Optoelectron. Engineering. 2013. V. 1. P. 19-27. 